\title{
Uncertainty and sensitivity analysis applied to a voltage series operational amplifier
}

\author{
Mohammad Nizam Ibrahim, Zainal Hisham Che Soh, Nor Shahanim Mohamad Hadis, Ali Othman \\ Fakulti Kejuruteraan Elektrik, UiTM Cawangan Pulau Pinang, Malaysia
}

\begin{tabular}{l} 
Article Info \\
\hline Article history: \\
Received Apr 24, 2020 \\
Revised Jun 23, 2020 \\
Accepted Jul 7, 2020 \\
\hline
\end{tabular}

\section{Keywords:}

Monte-carlo simulations

Non-inverting

Sensitivity analysis

Sobol' technique

Uncertainty analysis

\begin{abstract}
This paper investigates the applied uncertainty and sensitivity analysis to resistors used in a voltage series operational amplifier circuit. Two types of resistor bands are considered, namely the gold band (5\% tolerance) and the silver band ( $10 \%$ tolerance). To generate resistors uncertainty sample points, the SIMLAB uncertainty and sensitivity tool is used. A total of 6,144 sample points based on Sobol' technique has been created for each resistor band. The voltage series amplifier is modelled in MATLAB/Simulink. A MATLAB script has been written to execute Monte-Carlo simulations to read the resistor sample points, updating and executing the voltage series model and finally calculating the voltage gain. The result of uncertainty analysis shows that the produced voltage gain is uncertain within the range of \pm 1 for the gold band and \pm 2 for the silver band with respect to a target voltage gain. The result of the sensitivity analysis shows that each resistor, although their values are different, contributes equally to the uncertainty of the voltage gain.
\end{abstract}

This is an open access article under the CC BY-SA license.

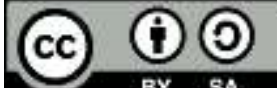

\section{Corresponding Author:}

Mohammad Nizam Ibrahim

Fakulti Kejuruteraan Elektrik

Universiti Teknologi MARA (UiTM) Cawangan Pulau Pinang

13500 Permatang Pauh, Pulau Pinang, Malaysia

Email:mnizam@uitm.edu.my

\section{INTRODUCTION}

Operational amplifier (op-amp) is a voltage controlled voltage source amplifier widely used in many electronic applications and circuits such as audio amplifier. The operation of a basic op-amp is to transform a low voltage signal to a higher voltage signal. The basic op-amp voltage gain without any feedback circuit is extremely high. The voltage gain (i.e. the ratio of output voltage to input voltage) can be between $20 \mathrm{~V} / \mathrm{mV}$ and $100 \mathrm{~V} / \mathrm{uV}$. As an example, a typical voltage gain for general purpose LM741 op-amp is $200 \mathrm{~V} / \mathrm{mV}$ [1]. The high voltage gain makes the basic op-amp component impractical for electronic applications. To overcome the problem, the op-amp is required to couple with a feedback circuit. The feedback circuit can reduce the extremely high voltage gain to a lower voltage gain that is usable in electronic applications.

There are two types of feedback circuits which are positive and negative feedback. The latter is commonly used due to several advantages such as stability at higher frequency, reduction of nonlinear distortion and bandwidth extension [2]. The negative feedback circuit can be built by adding a few external components such as resistors and capacitors. Figure 1 shows a simple non-inverting negative feedback circuit by using two external resistors $R_{1}$ and $R_{F}$ connected to a basic LM741 op-amp. This circuit, also known as a voltage series, can set its voltage gain target by choosing appropriate values for resistors $R_{1}$ and $R_{F}$. 


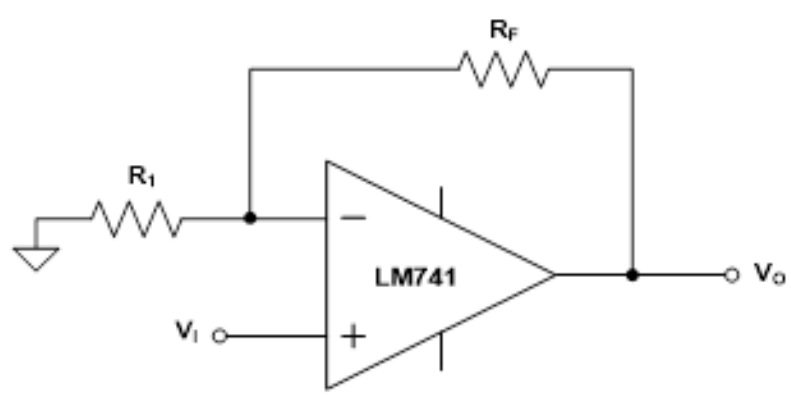

Figure 1. Voltage series op-amp

Theoretically, the voltage gain $A_{V}$ for the voltage series circuit is given by [2, 3]:

$$
\text { Voltage gain, } A_{V}\left(\frac{V}{V}\right)=1+\frac{R_{F}}{R_{1}}
$$

Using (1), a voltage gain of $11 \mathrm{~V} / \mathrm{V}$ can be achieved by using circuit resistors $R_{1}$ and $R_{F}$ of $1 \mathrm{k} \Omega$ and $10 \mathrm{k} \Omega$, respectively. The resulting voltage gain remains fixed at $11 \mathrm{~V} / \mathrm{V}$ providing that the resistors $R_{1}$ and $R_{F}$ values are ideal and unchanged. In practice, the resistors $R_{1}$ and $R_{F}$ are subjected to variations and errors due to the process of manufacturing and type of material used such as molded carbon composition and film resistors [4-7]. Resistor manufacturers provide resistor color code which includes information about resistor variations (i.e. tolerances). The resistor color code can be ranged from low precision (3-band) to high precision (6-band) [8-10]. In case of 3-band resistor, the tolerance band used is fixed at $20 \%$ of its nominal value. For other cases, the tolerance band is between $0.05 \%$ and $20 \%$. In any case, the tolerances indicate the possibility of resistor uncertainties which may also lead to voltage gain uncertainties. It is important to investigate the voltage gain uncertainties such that it is within certain design boundaries.

Uncertainty analysis can be used to assure that the voltage series circuit is designed to achieve a target voltage gain. Furthermore, it can also be used to show the bounds of circuit behavior. The uncertainty analysis quantifies all possible voltage gain outcomes that result from input uncertainties. The result of uncertainty analysis is the probability distributions of the voltage gain. In general, the small voltage gain uncertainty indicates the better voltage series circuit, and vice-versa. If the voltage gain uncertainty is high, some sensitive electronic components might be affected since these components work properly in a stable and fixed voltage gain condition. Beside the uncertainty analysis, it is also essential to apply the sensitivity analysis. Sensitivity analysis is how the input or model parameter uncertainty impacts the model outcome [11-13]. This means that the sensitivity analysis is much related to the uncertainty analysis. We can use the sensitivity analysis to quantify the contribution of each resistor to the uncertainty of the voltage gain. The result from the sensitivity analysis can be used to prioritize the circuit components [14]. A component with low sensitivity index (i.e. low contribution) can be set as a low prioritization component since it has a small effect on the voltage gain.

A few works have been carried out using uncertainty and sensitivity analysis techniques in an electronic field. W. Hernandez and J. de Vicente [15] investigated the uncertainty of the robust photometer circuit (RPC) transfer function. The paper shows an example of the design RPC where the gain and the phase shift were found to be robust. F. Yuan and A. Opal [16] proposed a new method for noise and sensitivity analysis of a multiphase periodically switched linear circuit. The study, however, has applied only the sensitivity analysis in the frequency domain. The results show that the output of the circuit is sensitive with respect to all elements of the circuit. J. Cheng et al. [17] have used the sensitive analysis to investigate the effects of electromagnetic interference (EMI) in the $\mu \mathrm{A} 741$ op-amp circuit. The result indicates that the differential input stage and the intermediate amplifying stage of the $\mu \mathrm{A} 741$ op-amp circuit are the most sensitive function model to the EMI effects. There has been also a study on a sensitivity analysis tool known as LIMSoft by N. B. Hamida, et al. [18]. The tool provides sensitivity computation and analysis to design fault-resistant circuits and generate test vectors. The recent study using the uncertainty analysis was conducted by T. Barić et al. [19]. The authors investigated the impact of balance resistor uncertainty on super-capacitors voltage and derived mathematical expression for the impact. It shows that the mathematical expression can be used to achieve the desired voltage variation level within safety margins.

In this paper, a Sobol' technique $[12,20]$ which is sampling-based has been applied to a model of a voltage series amplifier. The objective of this paper is to quantify the uncertainty and sensitivity of the 
voltage gain due to the uncertainty of its external resistors $R_{1}$ and $R_{F}$ shown in Figure 1 . Two common types of resistor bands which are gold $( \pm 5 \%$ tolerance) and silver $( \pm 10 \%$ tolerance $)$ are considered. The uncertainty and sensitivity analysis has been performed using SIMLAB [21] and MATLAB/Simulink tools [22]. SIMLAB is used to generate a sample of resistor uncertainties based on Sobol technique, measure voltage gain uncertainty and obtain sensitivity indices for resistors. The MATLAB/Simulink is used to model the simple voltage series amplifier and automate the execution of the model based on Monte-Carlo simulations.

\section{RESEARCH METHOD}

\subsection{Uncertainty and sensitivity analysis}

The aim of the uncertainty analysis is to quantify the propagation of inputs and parameters uncertainties to the model outputs, whereas the sensitivity analysis quantifies the contribution of those inputs and parameters to the uncertainties $[23,24]$. The uncertainty and sensitivity analysis can be broadly classified into local and global techniques. The local uncertainty and sensitivity analysis, such as Morris technique, is a simple technique whereby only one input factor is varied at a time. The input factor is varied around its nominal value while other factors being fixed at their nominal values. The local uncertainty and sensitivity analysis are suitable for screening important among many input factors. However, the local uncertainty and sensitivity analysis are unable to quantify the interaction contribution among input factors [11, 12, 20, 25].

In contrast, global uncertainty and sensitivity analysis, such as Sobol' technique, are based on varying all input factors simultaneously within their respective factor spaces. The advantage of the global analysis is in its ability to quantify the interaction contribution of input factors. The global analysis, however, requires a high computational cost [23]. In this paper, we use a Sobol' technique which is global uncertainty and sensitivity analysis. The Sobol' technique is a variance-based technique. This technique decomposes the output variance into components that quantify the importance of the single model input factors. The uncertainty and sensitivity analysis based on Sobol' technique have been demonstrated to be one of the most robust and popular techniques applied in many study areas [26-30].

Consider a mathematical model $f()$ which the output variable $Y$ is a nonlinear deterministic function of its $k$ input factors:

$$
Y=f\left(x_{1}, x_{2}, \ldots, x_{k}\right)
$$

where $x=\left[x_{1}, x_{2}, \ldots, x_{k}\right]$ are the uncertain input factors of the mathematical model. It is assumed that the input factors are random and independent variables. The output expected value $E(Y)$ and variance $V(Y)$ are given by:

$$
\begin{aligned}
& E(Y)=\int f\left(x_{1}, x_{2}, \ldots, x_{k}\right) d x \\
& V(Y)=\int\left[f\left(x_{1}, x_{2}, \ldots, x_{k}\right)-E(Y)\right]^{2} d x
\end{aligned}
$$

The expected value of $Y$ conditional on $x_{i}$ is given by:

$$
E\left(Y \mid x_{i}\right)=\int f\left(x_{1}, x_{2}, \ldots, x_{k}\right) \frac{d x}{d x_{i}}
$$

The first order sensitivity index of the model is defined by:

$$
S_{i}=\frac{V\left[E\left(Y \mid x_{i}\right)\right]}{V(Y)}
$$

The total order sensitivity index of the model is defined by:

$$
S_{T i}=1-\frac{V\left[E\left(Y \mid x_{-i}\right)\right]}{V(Y)}
$$

Where $x_{-i}$ represents the total of input factors excluding $x_{i}$.

The first order index shows the effect of a single parameter on the output variance. The total order index shows the effect of the first order and interaction involving that parameters (second and higher order effects) on the output variance. If the first order and total order indices are similar, the model has mostly no interaction effects $[11,12]$. To assist in computing the $S_{i}$ and $S_{T i}$ indices, the SIMLAB uncertainty and 
sensitivity tool is used. The SIMLAB is a software tool specifically designed for investigating uncertainty and sensitivity analysis [21].

\subsection{Methodology}

The methodology used to apply uncertainty and sensitivity analysis is shown in Figure 2. It is implemented within two environments which are the SIMLAB and the MATLAB/Simulink. The function of the SIMLAB is for pre-processing and post-processing by generating sample points and finally calculating uncertainty and sensitivity indices. The MATLAB/Simulink is used to simulate a voltage series model and calculate its voltage gain.

Firstly, two input factors, $R_{1}$ and $R_{F}$, defined their parameters and uncertainties in the SIMLAB environment. There were two study cases since we considered two resistor tolerance bands $( \pm 5 \%$ and $\pm 10 \%)$. Table 1 summarizes the investigated input factors with their corresponding uncertainties.

Table 1. Input factors uncertainty

\begin{tabular}{ccc}
\hline Input & Case I $( \pm 5 \%)$ & Case II $( \pm 10 \%)$ \\
\hline$R_{1}$ & $1 k \Omega \pm 5 \%$ & $1 k \Omega \pm 10 \%$ \\
$R_{F}$ & $10 k \Omega \pm 5 \%$ & $10 k \Omega \pm 10 \%$ \\
\hline
\end{tabular}

For both case I and case II, the probability of each resistor was assumed to be a uniform distribution. This means that the probabilities for the occurrence of these resistor values within their possible ranges are the same. Based on Sobol' sensitivity technique selected in the SIMLAB tool, each case study has generated $6144 \times 2$ uncertain sample points.

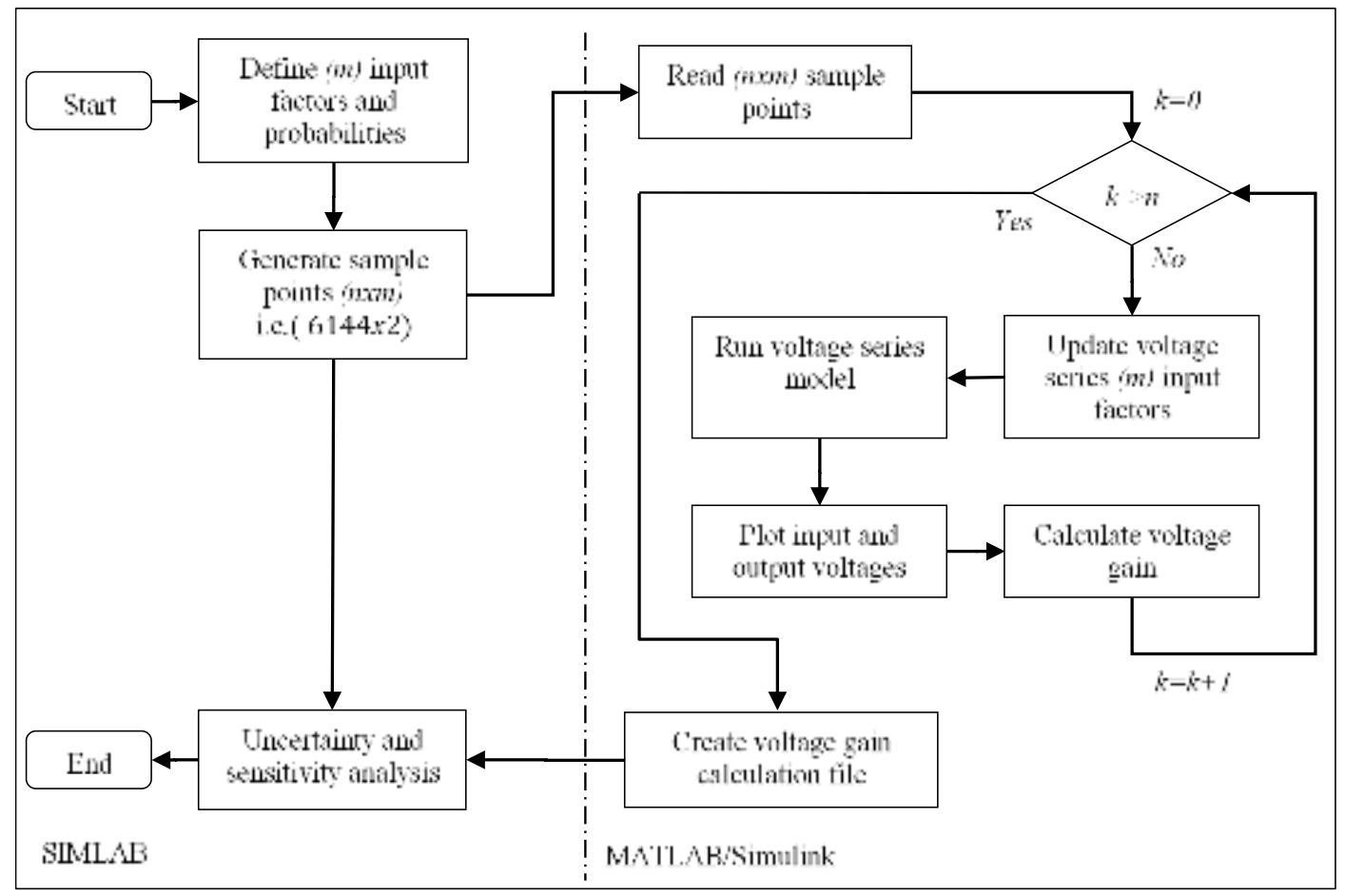

Figure 2. Uncertainty and sensitivity analysis methodology

In the second environment, we initially modelled a voltage series in Simulink as shown in Figure 3. We set $R_{1}$ and $R_{F}$ as the variable input factors such that these factors would be sequentially updated when executing the model. We used MATLAB scripts to automatically read the $6144 \times 2$ sample points and for each row of the sample points, the scripts updated the variable input resistors, $R_{1}$ and $R_{F}$. Then, the voltage series model was executed for 5 milliseconds. The peak to peak input $V_{i(p p)}$ and output peak to peak voltage $V_{o(p p)}$ were automatically captured. The voltage gain was calculated using (8): 


$$
\text { Voltage gain, } A_{V}\left(\frac{V}{V}\right)=\frac{V_{o(p p)}}{V_{i(p p)}}
$$

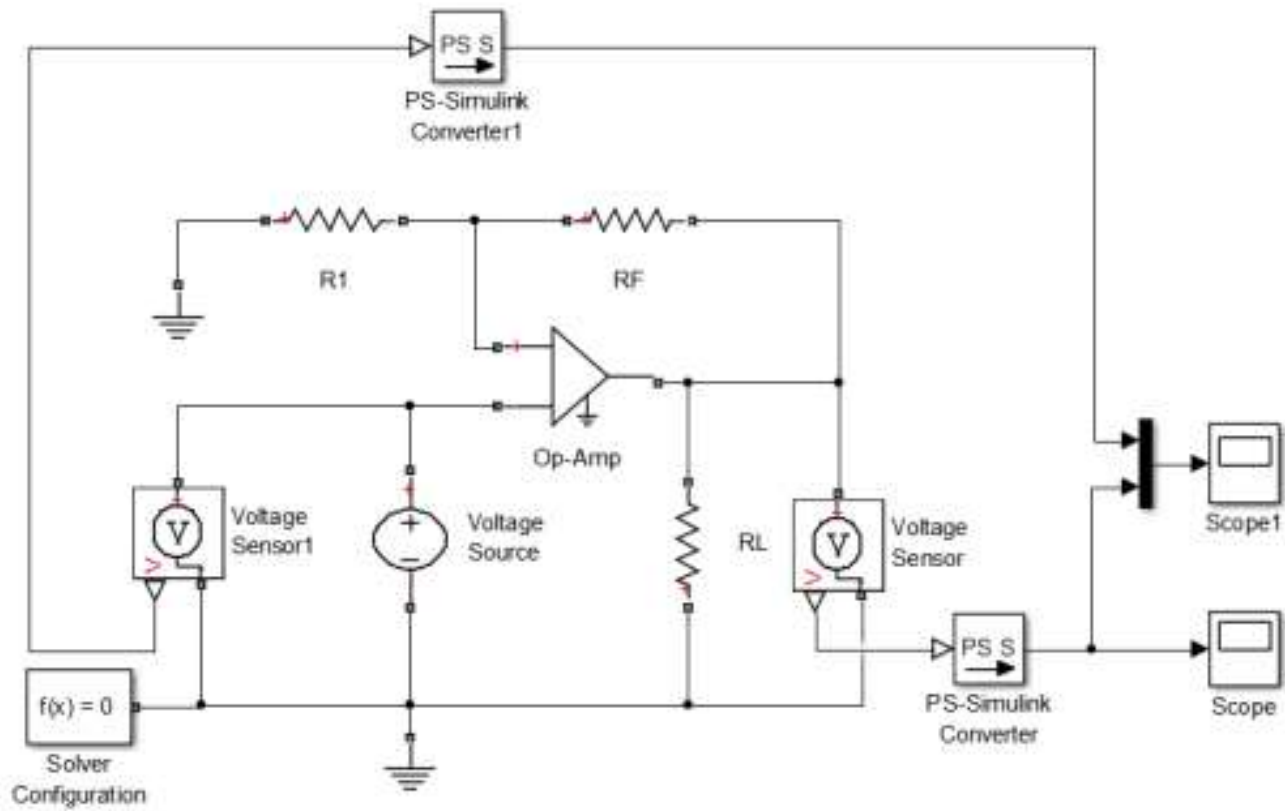

Figure 3. Voltage series model in simulink

The process to update $R_{1}$ and $R_{F}$, runs the voltage series model, plots the input and output voltages and calculates the voltage gain based on Monte-Carlo simulations. The process has been repeating for all 6144 sample points. Once completed, a file containing the list of calculated voltage gain was created. Finally, the file containing the list of voltage gain and the sample points were imported into SIMLAB again for post-processing. The post-processing includes the mapping between the generated sample points and their corresponding voltage gain based on the Sobol' technique. The results of uncertainty and sensitivity analysis are described next.

\section{RESULTS AND DISCUSSION}

There are two case studies which are voltage series that used resistors with $\pm 5 \%$ tolerance (case I) and resistors with $\pm 10 \%$ tolerance (case II). For both cases, the uncertainty and sensitivity analysis based on Sobol' technique has been applied to quantify the uncertainty of voltage gain and the contribution of the resistors to the uncertainty of the voltage gain.

\subsection{Case I (Resistors with $\pm \mathbf{5} \%$ tolerance)}

This case assumes that the resistors $R_{1}$ and $R_{F}$ are uncertain within $\pm 5 \%$ from their nominal value. Thus, the range values for the resistors $R_{1}$ and $R_{F}$ are $0.95 k \Omega \leq R_{1} \leq 1.05 k \Omega$ and $9.5 k \Omega \leq R_{F} \leq 10.5 k \Omega$, respectively. Using the SIMLAB tool, a total of 6144 sample points has been generated as shown by Figure 4. The minimum value for $R_{1}$ is $0.95 \mathrm{k} \Omega$ and the maximum value is $1.05 \mathrm{k} \Omega$. Similarly, the minimum and maximum values for $R_{F}$ are $9.5 \mathrm{k} \Omega$ and $10.5 \mathrm{k} \Omega$, respectively. The distributions for both $R_{1}$ and $R_{F}$ sample points are distributed in a uniform way.

As an example, Figure 5 shows a selected sample set out of 6144 sample sets used to simulate the input and output voltages. The selected sample set values are $R_{1}=0.9569 \mathrm{k} \Omega$ and $R_{F}=9.9920 \mathrm{k} \Omega$. The simulation shows that the input peak to peak voltage, $V_{i(p p)}=2 \mathrm{mV}$ while the output peak to peak voltage, $V_{o(p p)}=23.02 \mathrm{mV}$. Therefore, the voltage gain, $A_{v}=23.02 \mathrm{mV} / 2 \mathrm{mV}=11.51$. As the target voltage gain is 11 , the produced voltage gain is deviated by $=(11.51-11)=0.51$.

Figure 6 shows the histogram of the voltage gain that has been tabulated for all the 6144 sample points. Ideally, with $R_{1}=1 \mathrm{k} \Omega$ and $R_{F}=10 \mathrm{k} \Omega$, the target voltage gain is 11 . However, with the $5 \%$ uncertainty for both resistors $R_{1}$ and $R_{F}$, the minimum and maximum possible values for voltage gain are

Uncertainty and sensitivity analysis applied to a voltage series operational ... (Mohammad Nizam Ibrahim) 
10.1 and 12.1., respectively. Thus, the range of voltage gain uncertainties is between 10.1 and 12.1. This result indicates that the $5 \%$ uncertainty for both resistors $R_{1}$ and $R_{F}$ resulting in the voltage gain is uncertain, which is approximately $11 \pm 1$.

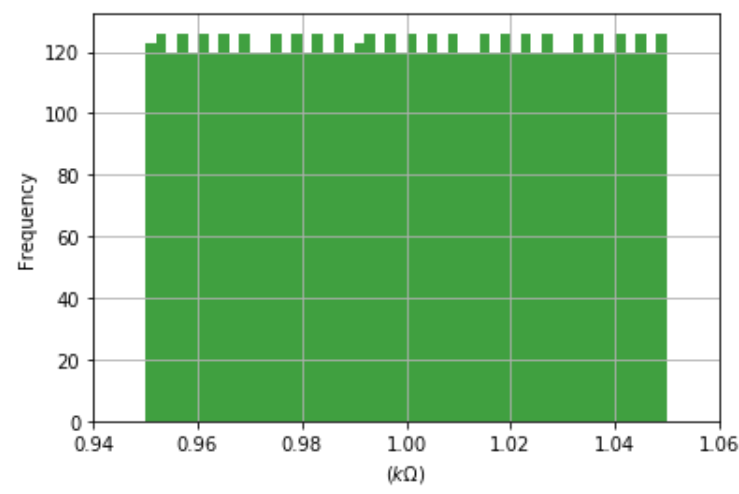

(a) Resistor $R_{1}$

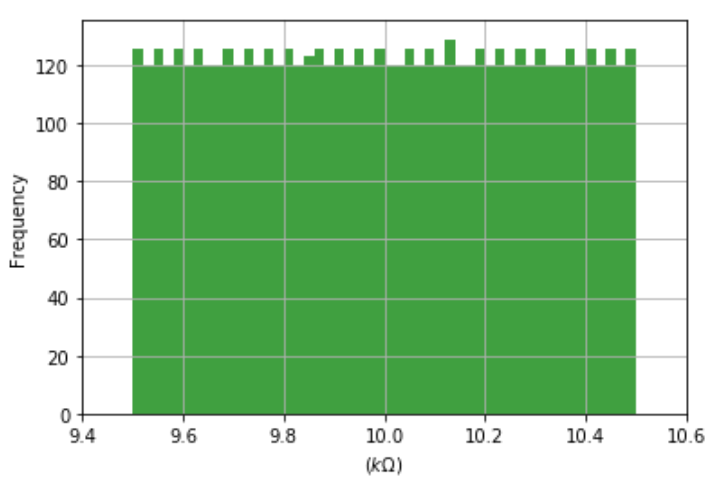

(b) Resistor $R_{F}$

Figure 4. Resistor $R_{1}$ and $R_{F}$ histogram

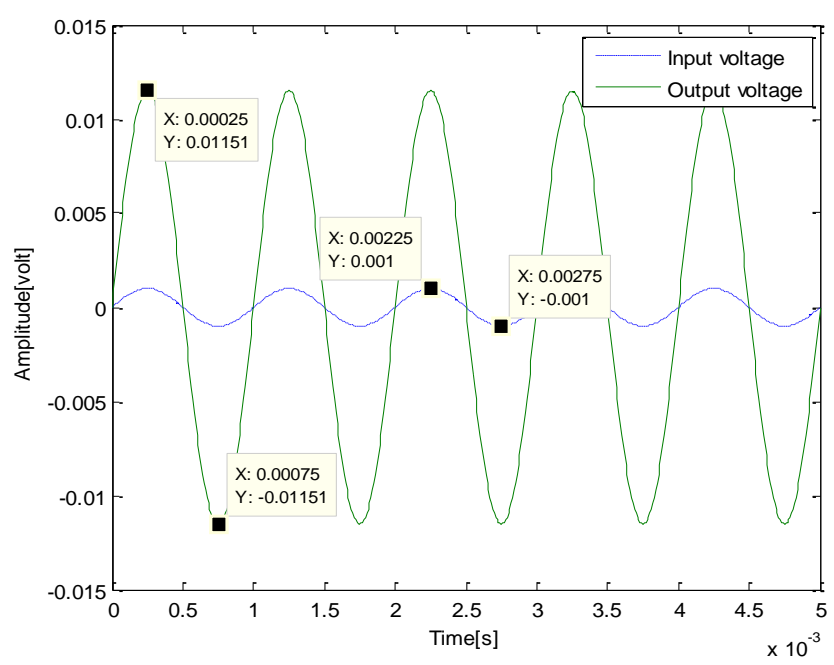

Figure 5. Input and output voltages of voltage series for $R_{1}=0.9569 \mathrm{k} \Omega, R_{F}=9.9920 \mathrm{k} \Omega$

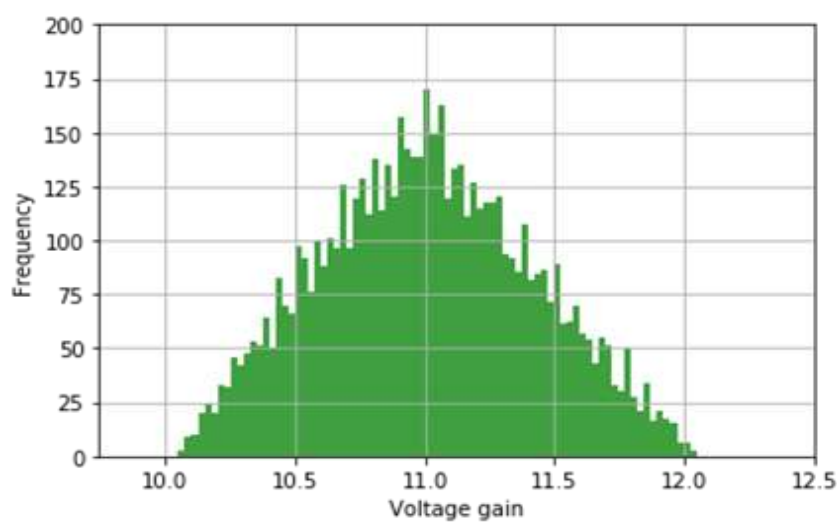

Figure 6. Voltage gain histogram (case I) 
Table 2 shows the sensitivity indices for case I. The first order index for $R_{1}$ and $R_{F}$ is 0.500578 and 0.499302 , respectively. Since the resistor indices are almost of the same values, their contribution level to the voltage gain uncertainty is similar. This result means that although the $R_{F}$ is 10 times the $R_{1}$ values, these resistors make an equally important input factor for the voltage series circuit. $R_{1}$ and $R_{F}$ total order indices also show a similar value as compared to their first order indices which means that no interaction between $R_{1}$ and $R_{F}$ contributes to the voltage gain uncertainty.

Table 2. Sensitivity indices for $R_{1}$ and $R_{F}$ (Case I)

\begin{tabular}{ccc}
\hline Resistor & First order & Total order \\
\hline$R_{1}$ & 0.500578 & 0.501015 \\
$R_{F}$ & 0.499302 & 0.499740 \\
\hline
\end{tabular}

\subsection{Case II (Resistors with $\pm \mathbf{1 0} \%$ tolerance)}

This case is like case I except that the resistors $R_{1}$ and $R_{F}$ are uncertain at wider ranges. In this case, both the $R_{1}$ and $R_{F}$ are uncertain at $\pm 10 \%$ from their nominal values. Figure 7 shows the histogram of voltage gain for case II. The ideal voltage gain is 11 . However, with the uncertainty of both resistors $R_{1}$ and $R_{F}$ the voltage gain is uncertain between 9.2 and 13.2. This result indicates that the voltage gain is uncertain which is approximately $11 \pm 2$.

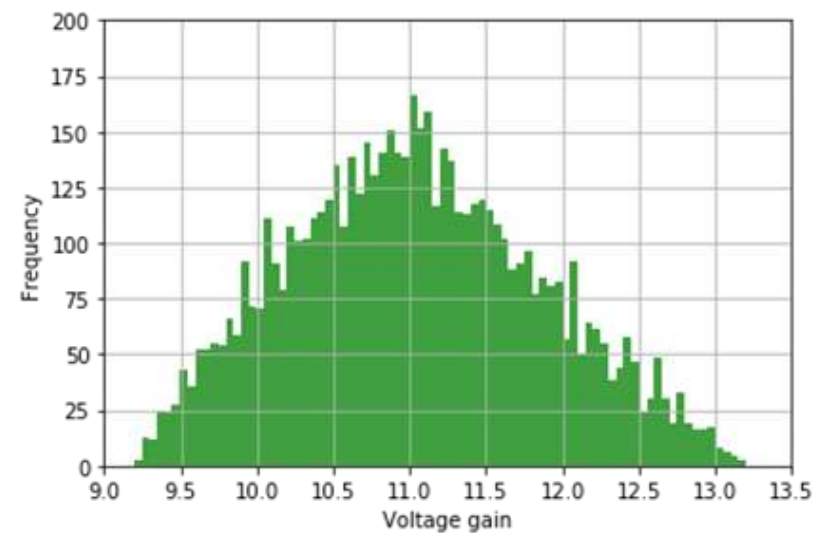

Figure 7. Voltage gain histogram (case II)

Table 3 tabulates the sensitivity indices for case II. The first order indices for $R_{1}$ and $R_{F}$ are 0.501499 and 0.497151 , respectively. This result shows that $R_{1}$ and $R_{F}$ index values are almost the same indicating that their contribution level to the voltage gain uncertainty is similar. The $R_{1}$ and $R_{F}$ total order indices also show almost similar value as compared to their first order indices. This means that no interaction between $R_{1}$ and $R_{F}$ contributes to the voltage gain uncertainty

Table 3. Sensitivity indices for $R_{1}$ and $R_{F}$ (case II)

\begin{tabular}{ccc}
\hline Resistor & First order & Total order \\
\hline$R_{1}$ & 0.501499 & 0.503216 \\
$R_{F}$ & 0.497151 & 0.498868 \\
\hline
\end{tabular}

\section{CONCLUSION}

This paper shows the applied uncertainty and sensitivity analysis based on Sobol's technique to the voltage series amplifier. The uncertainty analysis is used to measure the uncertainty of voltage gain due to the uncertainties in the resistors of the amplifier. Furthermore, the sensitivity analysis is used to identify the contribution of the resistors to the uncertainty of the voltage gain. Two cases of resistor uncertainties are considered which are resistor with a gold tolerance (case I) and resistor with a silver tolerance (case II). For both cases, the ideal target voltage gain has been set to 11 . The results for the case I show that the voltage 
gain shows a small uncertainty which is between 10.1 and 12.1. As expected, the voltage gain shows a wider uncertainty for case II since the resistors with low tolerance are used. The uncertainty of voltage gain for case II is between 9.2 and 13.2. The applied sensitivity analysis in both cases shows that voltage series resistors $R_{1}$ and $R_{F}$ contribute equally to the output voltage uncertainty although the $R_{F}$ value is larger than $R_{1}$ by a factor of 10 . The resistors $R_{1}$ and $R_{F}$, however, show no contribution of interaction on the uncertainty of the voltage gain. Future works will consider electronic circuits of higher complexities.

\section{REFERENCES}

[1] Available online: http://pdf.datasheetcatalog.com/datasheet/fairchild/LM741.pdf (last accessed October 2020).

[2] M. H. Rashid, "Microelectronic Circuit: Analysis and Design," Cengage Learning, 3rd. Edition, 2016.

[3] B. Carter, "Op Amps for Everyone," Newnes, 5th. Edition, 2017.

[4] M. Schenk, et al, "Worst-case analysis of electronic circuits based on an analytic forward solver approach," COMPEL-The international journal for computation and mathematics in electrical and electronic engineering, vol. 38, no. 5, pp. 1655-1666, 2019.

[5] J. Hlina et al., "Reliability of printed power resistor with thick-film copper terminals," Microelectronic Engineering, vol. 216, 2019.

[6] P. Billa, Y. Meihru, and Y. S. Rao, "Studies on the Thermal Variations of Resistance of PVC-Graphite Thick Film Resistors," Materials Today: Proceedings, vol. 11, pp. 1010-1014, 2019.

[7] E. D. Gates, "Introduction to Electronics," Cengage Learning, 6th. Edition, 2011.

[8] Available online: https://www.electronics-tutorials.ws/resistor/res_2.html (last accessed October 2020).

[9] Available online: https://www.electrical4u.com/resistor-color-coding (last accessed October 2020).

[10] A. Kagan, "Excel by Example: Example 8-Resistor Color Code Decoder Using Speech Input," Newnes, pp. 127$142,2004$.

[11] A. Saltelli, et al., "Global Sensitivity Analysis. The Primer," John Wiley \& Sons Ltd, 2008.

[12] E. Borgonovo and E. Plischke, "Sensitivity analysis: A review of recent advances," European Journal of Operational Research, vol. 248, issue 3, pp. 869-887, 2016.

[13] A. Saltelli, et al., "Why so many published sensitivity analyses are false: A systematic review of sensitivity analysis practices," Environmental Modelling \& Software, vol. 114, pp. 29-39, 2019.

[14] Q. Xiong et al., "Parameter importance ranking for SBLOCA of CPR1000 with moment-independent sensitivity analysis," Nuclear Engineering and Technology, vol. 52, no. 12, pp. 2821-2835, 2020.

[15] W. Hernandez and J. D. Vicente, "Measurement uncertainty estimation of a robust photometer circuit," Sensors (Basel), vol. 9, no. 4, pp. 3149-3160, 2009. doi:10.3390/s90403149.

[16] F. Yuan and A. Opal, "Noise and sensitivity analysis of periodically switched linear circuits in frequency domain," in IEEE Transactions on Circuits and Systems I: Fundamental Theory and Applications, vol. 47, no. 7, pp. 986998, July 2000. DOI:10.1109/81.855454.

[17] J. Cheng et al., "Sensitive analysis of EMI effect in the $\mu \mathrm{A} 741$ operational amplifier circuit," 2013 International Conference on Quality, Reliability, Risk, Maintenance, and Safety Engineering (QR2MSE), pp. 1118-1121, 2013. doi: 10.1109/QR2MSE.2013.6625762.

[18] N. B. Hamida, et al., "LIMSoft: automated tool for design and test integration of analog circuits," Proceedings International Test Conference 1996, pp. 571-580, 1996.

[19] T. Barić, et al, "Impact of balance resistor uncertainty on voltages across supercapacitors," Journal of Energy Storage, vol. 22, pp. 131-136, 2019.

[20] H. M. Wainwright et al., "Making sense of global sensitivity analyses," Computers \& Geosciences, vol. 65, pp. 8494, 2014.

[21] SimLab, "Software package for uncertainty and sensitivity analysis," 2011. Available: https://ec.europa.eu/jrc/en/samo/simlab (last accessed October 2020).

[22] MATLAB: http://www.mathworks.com/

[23] A. Saltelli, K. Chan, and M. Scott (Eds.), "Sensitivity Analysis," John Wiley \& Sons Publishers, New York, 2008.

[24] L. Rivalin et al., "A comparison of methods for uncertainty and sensitivity analysis applied to the energy performance of new commercial buildings," Energy and Buildings, vol. 166, pp. 489-504, 2018.

[25] S. Xiao, Z. Lu, and L. Xu,, "A new effective screening design for structural sensitivity analysis of failure probability with the epistemic uncertainty," Reliability Engineering \& System Safety, vol.156, pp. 1-14, 2016.

[26] A. Bouloré, C. Struzik, F. Gaudier, "Uncertainty and sensitivity analysis of the nuclear fuel thermal behavior," Nuclear Engineering and Design, vol. 253, pp. 200-210, 2012.

[27] A. Ferrari, S. Gutiérrez and G. Sin, "Modeling a production scale milk drying process: parameter estimation, uncertainty and sensitivity analysis," Chemical Engineering Science, vol. 152, pp. 301-310, 2016.

[28] L. Bouazizi, et al., "Uncertainty and sensitivity analysis of porous materials acoustic behavior," Applied Acoustics, vol. 144, pp. 64-70, 2019.

[29] Y. Gan et al., "A comprehensive evaluation of various sensitivity analysis methods: A case study with a hydrological model," Environ. Model. Softw., vol. 51, pp. 269-285, 2014.

[30] C. Zhang, J. Chu and G. Fu, "Sobol's sensitivity analysis for a distributed hydrological model of Yichun River Basin, China," J. Hydrol., vol. 480, pp. 58-68, 2013. 


\section{BIOGRAPHIES OF AUTHORS}

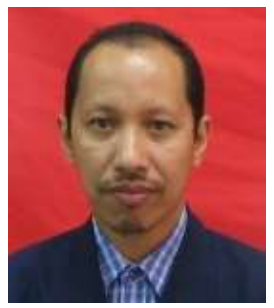

Mohammad Nizam bin Ibrahim is a senior lecturer at the Faculty of Electrical Engineering, Universiti Teknologi MARA (UiTM) Cawangan Pulau Pinang. He obtained his B. Eng (Hons) in Electronic Engineering from University of Portsmouth, UK in 1997, MSc. in Electronic System Design Engineering from Universiti Sains Malaysia in 2004 and $\mathrm{PhD}$ in Electrical from University of Adelaide, Australia in 2012. His main interests are transmission lines protection, sensitivity and uncertainty analysis, fault monitoring and simulation and web applications.

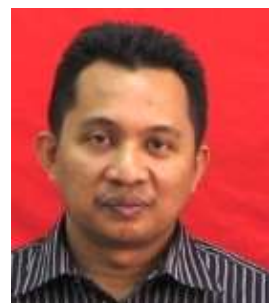

Zainal Hisham bin Che Soh was born in Machang, Kelantan on 16th March 1974. He obtained his B. Eng (Hons) in Electronic Engineering from University of Leeds, UK in 1997, MSc. In Computer Science in Real-Time Software Engineering from UTM in 2004 and PhD in Electrical and Electronic from USM in 2013. His research interest in Internet of Things, Big Data, Distributed/Parallel Computing, Artificial Intelligence, Microcontroller System and Wireless Sensor Network. He is recognized as Graduate Technologist and Professional Technologist by the Malaysia Board of Technologists (MBOT). He works in UiTM Pulau Pinang under Faculty of Electrical Engineering, UiTM, Pulau Pinang. He is a member of IEE, IET, BEM and MySEIG.

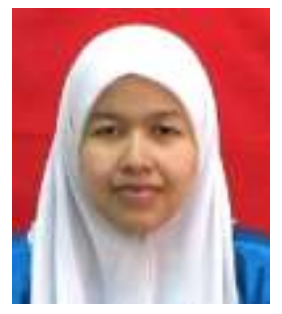

Nor Shahanim binti Mohamad Hadis is a senior lecturer at the Faculty of Electrical Engineering, Universiti Teknologi MARA (UiTM) Cawangan Pulau Pinang. She obtained her Diploma in Electrical (Electronics) Engineering from Universiti Teknologi MARA in 2002, Bachelor (Hons) in Electrical Engineering from Universiti Teknologi MARA in 2005, MSc. in Microelectronic from Universiti Kebangsaan Malaysia in 2006 and PhD in Nano-Material \& Device Fabrication from University Sains Malaysia in 2018. Her main interests are microelectronics device, device fabrication and characterization, bio-sensor, memristor and Internet of Things.

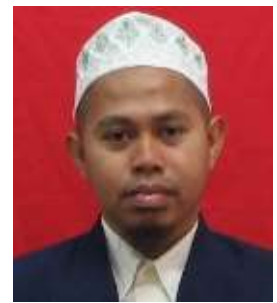

Ali Othman obtained BEng (Hons) degree in Electrical and Electronic Engineering from the University of Bradford, United Kingdom in 1997, the MSc degree in Digital Communications from the University of Bath, United Kingdom in 2003 and PhD in Antenna and Propagation from Universiti Sains Malaysia, Malaysia in 2014. He is currently a Senior Lecturer at the Faculty of Electrical Engineering, Universiti Teknologi MARA Pulau Pinang. His research interest includes antenna and propagation, dielectric resonator antenna, microstrip antenna and wireless communications. 